\title{
Letter to the Editor: Determination of Brain Type Creatine Kinase for Diagnosis of Perinatal Asphyxia-Choice of Method
}

\author{
JOE J. HOO AND H. WERNER GOEDDE
}

Institute of Human Genetics, University of Hamburg, West Germany

The paper of Cuetas (Creatine Kinase Isoenzymes in High-Risk Infants, Pediatr. Res., 14: 935 1980) has confirmed the results of earlier studies stating that severe asphyxia at birth was associated with elevation of brain type creatine kinase (CK-BB) in either the serum (2) or in the cerebrospinal fluid (8); thus, the determination of $\mathrm{CK}-\mathrm{BB}$ in the serum could be used as a laboratory adjunct to the diagnosis of severe cerebral hypoxia, which is made hitherto almost entirely on the basis of clinical evaluation.

Nevertheless, we have some doubt on the data regarding the absolute CK-BB activity presented in the paper of Cuetas, mainly because the data was obtained by a method of electrophoretic separation. As it will be discussed below, the method of electrophoretic separation is a questionable procedure for this purpose.

In 1973, Frotscher et al. (5) reported a change of electrophoretic mobility of rabbit brain $\mathrm{CK}-\mathrm{BB}$ following incubation in human serum at $37^{\circ} \mathrm{C}$. Without prior incubation, CK-BB migrated rapidly toward the anode and formed the most anodic band; however, after incubation at $37^{\circ} \mathrm{C}$ a part or all of the CK-BB migrated only as far as $\mathrm{CK}-\mathrm{MB}$, which appeared between the bands of $\mathrm{CK}-\mathrm{BB}$ and CK-MM. Later, Cho et al. (3) and Cho and Meltzer (4) confirmed this phenomenon on human brain CK-BB. They further showed that the longer the incubation, the slower the mobility of CK-BB. By means of an immunoprecipitation method Bayer et al. (1) were able to prove that this electrophoretically altered CK$\mathrm{BB}$ was not a newly hybridized CK-MB. Accompanying this change of electrophoretic mobility a tremendous decay of the catalytic activity of CK-BB was also noted. Indeed, a very short half life of CK-BB in the blood circulation has also been suggested and a half life of $3 \mathrm{~h}$ or even shorter has recently been estimated (7).

It is therefore very likely that CK-BB could undergo similar changes when circulating in the blood stream. After its influx into the blood circulation, CK-BB would soon experience alteration of its electrophoretic mobility and decay of its catalytic activity. By means of an electrophoretic separation, an unknown amount of $\mathrm{CK}-\mathrm{BB}$ would be mistaken as CK-MB. Consequently, the calculated $\mathrm{CK}-\mathrm{BB}$ activity according to the densitometric measurement would no longer represent the actual measurable CK-BB activity. The rapid decrease of $\mathrm{CK}-\mathrm{BB}$ activity accompanied with an increase of CK-MB and CK-MM activities in the first few hours after birth, as stated by Cuetas, could be in part explained by the above mentioned phenomenon.

Immunologic methods for quantitative determination of $\mathrm{CK}$ isoenzymes may provide more accurate results. The immunoprecipitation method (9) with some modifications i.e., using only onetenth of the sample and reagents described as well as omitting the $1 \mathrm{~h}$ incubation at $37^{\circ} \mathrm{C}$, is presently applied in our laboratory and would not be affected by a change of electrophoretic mobility and could be used for nursery patients as well. Only $0.25 \mathrm{ml}$ plasma is needed for one determination; however, the immunoprecipitation method only measures the catalytic activity of CK-BB, which still remains active at the moment of blood drawing. In case that the influx of CK-BB into the circulation had ceased to occur some time before the blood was drown, the actual amount of CK-BB would also be missed owing to the rapid decay of its catalytic activity.

In this respect, the method of radioimmunoassay, which measures the mass concentration of CK-BB independent of its catalytic activity $(6,10)$, appears to provide a further alternative to the method of immunoprecipitation. Nonetheless, this determination of mass concentration of $\mathrm{CK}-\mathrm{BB}$ is not free from any shortcoming neither, for instance, one would still not be able to differentiate maternal uterine CK-BB from the brain CK-BB of the newborn, especially as long as the clearance rate of CK-BB remains unknown.

The results of Cuetas are indeed encouraging. A laboratory documentation of cerebral hypoxia would be very useful, among others for later assessment of neurologic and mental handicaps, especially in regard to genetic counselling, as genetic form of psychomotor retardation has to be separated from brain damage due to hypoxia. Certainly, more studies have still to be done to find out the most reliable as well as simple method for the determination of CK-BB.

\section{REFERENCES AND NOTES}

1. Bayer, P. M., Gergely, Th., Gabl, F. and Deutsch, E.: Serum-induced changes in electrophoretic mobility of creatine phosphokinase isoenzymes? Clin. Chim. Acta, 79: 261 (1977).

2. Becker, M. and Menzel, K.: Brain-typical creatine kinase in the serum of newborn infants with perinatal brain damage. Acta Paediatr. Scand., 67: 177 (1978)

3. Cho, H. W., Meltzer, H. Y., Joung, J. and Goode, D.: Effect of incubation in human plasma on electrophoretic mobility of brain-type creatine phosphokinase. Clin. Chim. Acta, 73: 257 (1976)

4. Cho, H. W. and Meltzer, H. Y.: Factors affecting stability of isozymes of creatine phosphokinase. Amer. J. Clin. Path., 71: 75 (1979).

5. Frotscher, U., Dominik, B., Richter, R., Zschaege, B., Schulte-Lippern, M Jenett, G., Messerschmidt, W., Schmidtmann, W. and Wilbrandt, R.: Die Instabilität der Kreatin-Phosphokinase-Isoenzyme im Serum. Klin. Wschr., 51: 801 (1973)

6. Homburger, H. A. and Jacob, G. L.: Creatine kinase radioimmunoassay and isoenzyme electrophoresis compared in the diagnosis of acute myocardial infarction. Clin. Chem., 26: 861 (1980).

7. Lang, H.: The creatine Kinase BB isoenzyme. In: Lang, H. (ed.) Creatine kinase isoenzymes. Pathophysiology and Clinical Application. (Springer-Verlag, Berlin-Heidelberg-New York, New York 1981).

8. Meberg, A., Hetland, Ö., Sommer, F. and Vaagenes, P.: Creatine kinase in cerebrospinal fluid in newborn infants. Clin. Chim. Acta, 85: 95 (1978)

9. Wũrzburg, U., Hennrich, N., Orth, H. D., Lang, H., Prellwitz, W., Neumeier, D., Knedel, $M$. and Rick, W.: Quantitative determination of creatine kinase isoenzyme catalytic concentrations in serum using immunological methods. $\mathbf{J}$. Clin. Chem. Clin. Biochem., 15: 131 (1977).

10. Van Steirteghem, A. C., Robertson, E. A., and Zweig, M. H.: Distribution of serum concentrations of creatine kinase $\mathrm{MM}$ and $\mathrm{BB}$ isoenzymes measured by radioimmunoassay. Clin. Chim. Acta, 93: 25 (1979). 